\title{
Affine Tensor Product Model Transformation
}

\author{
József Kuti $(\mathbb{D})$ and Péter Galambos \\ Antal Bejczy Center for Intelligent Robotics, Óbuda University, Bécsi út 96/b, Budapest 1034, Hungary \\ Correspondence should be addressed to József Kuti; jozsef.kuti@irob.uni-obuda.hu
}

Received 26 October 2017; Accepted 27 December 2017; Published 20 March 2018

Academic Editor: Eulalia Martínez

Copyright (C) 2018 József Kuti and Péter Galambos. This is an open access article distributed under the Creative Commons Attribution License, which permits unrestricted use, distribution, and reproduction in any medium, provided the original work is properly cited.

\begin{abstract}
This paper introduces the novel concept of Affine Tensor Product (TP) Model and the corresponding model transformation algorithm. Affine TP Model is a unique representation of Linear Parameter Varying systems with advantageous properties that makes it very effective in convex optimization-based controller synthesis. The proposed model form describes the affine geometric structure of the parameter dependencies by a nearly minimum model size and enables a systematic way of geometric complexity reduction. The proposed method is capable of exact analytical model reconstruction and also supports the samplingbased numerical approach with arbitrary discretization grid and interpolation methods. The representation conforms with the latest polytopic model generation and manipulation algorithms. Along these advances, the paper reorganizes and extends the mathematical theory of TP Model Transformation. The practical merit of the proposed concept is demonstrated through a numerical example.
\end{abstract}

\section{Introduction}

The importance of polytopic system descriptions is beyond doubt since the development of influential polytopic modelbased analysis and synthesis methods initially introduced by Boyd et al. in [1]. These approaches offer a simple way for stability verification and robust or gain-scheduling controller design via Linear Matrix Inequality (LMI) based methods for polytopic Linear Parameter Varying (LPV) and quasi-LPV (qLPV) models.

TP Model Transformation was introduced as a numerical approach to constructing polytopic TP forms of LPV/qLPV models [2] serving as an alternative to analytical procedures such as the sector nonlinearity technique [3]. Furthermore, the separated parameter dependencies within the TP structure can be exploited during the controller design extending the polytopic model-based control analysis and synthesis methods $[4,5]$.

In the past decade, TP Model Transformation has been matured and became an extensive framework within polytopic model-based control $[2,3,6]$. Former related works (e.g., [7-11]) obtained the polytopic TP Model through the HOSVD-based intermediate TP form [12], although the resulting polytopic model does not really benefit from the properties of the HOSVD-based form such as complexity reduction capability and uniqueness.

A recent paper of the authors [13] established the affine geometric background of polytopic TP Model generation and proposed a direct way to determine the polytopic structures. First, it obtained the affine hulls of the subtensors of the discretized tensor and then the enclosing polytopes were established on the affine subspaces.

The paper proposes the Affine TP Model that substantially improves the polytopic TP Model generation and manipulation methodology by combining the affine geometric interpretations [13] with the benefits of higher-order SVD (HOSVD) based TP Model [2, 12, 14].

Consolidating the affine geometry-based approach, the main contribution of this paper is the introduction of a new intermediate TP Model (like the HOSVD-based form) that provides a unique description of affine geometric properties serving as direct input for polytopic model construction methods (see [13, 15-17]). Furthermore, it reserves all the benefits of the HOSVD-based form: similar uniqueness, compact representation, and capability of complexity reduction. We refer to the new intermediate form as Affine TP Model. 
The next section discusses the abbreviations and notations used in the paper. Section 3 recalls some concepts of tensor algebra related to polytopic TP modeling; then Section 4 discusses the polytopic form of univariate functions showing its relevance to affine geometrics and introduces the affine SVD. In Section 5, affine SVD is applied to obtain the Affine TP Model. Section 6 describes the application to generate and manipulate polytopic TP Models; then Section 7 shows a simple numerical example. Finally, Section 8 concludes the paper.

\section{Notations}

The following abbreviations and notations are used within this paper:

(q)LPV: (quasi)Linear Parameter Varying

LMI: Linear Matrix Inequality

SVD: Singular Value Decomposition

HOSVD: higher-order singular value decomposition

TP Model: Tensor Product Model

$a, A, b, B, \ldots$ scalar values

$\mathbf{a}, \mathbf{b}, \ldots .:$ vectors

A, B, ...: matrices

$H$ : a Hilbert space, in general

$\mathfrak{a}, \mathfrak{b}, \ldots$ : elements of $H$, in general

$\mathbf{0}^{a \times b}, \mathbf{1}^{a \times b}: a \times b$ size matrix of zeros/ones

$\mathbf{E}^{a \times b}: a \times b$ size identity matrix

$\delta_{i j}$ : dirac-delta $\left(\delta_{i i}=1, \delta_{i j}=0\right.$ if $\left.i \neq j\right)$

$\mathfrak{A}, \mathfrak{B}, \ldots$ sets on $\mathbb{R}^{a}, H, \ldots$

$\mathscr{A}, \mathscr{B}, \ldots$. tensors

$\mathbf{A}_{(n)}:$ n-mode unfold matrix of tensor $\mathscr{A}$

$\mathbf{A}^{(n)}, \mathscr{B}^{(n)}$ : indexing of different matrices, tensors

$\mathscr{A} \times{ }_{n} \mathrm{U}: n$-mode tensor product

$\mathscr{A} \underset{n=1}{\stackrel{N}{\otimes}} \mathbf{U}^{(n)}:$ multiple tensor product as $\mathscr{A} \times{ }_{1} \mathbf{U}^{(1)} \cdots \times_{N} \mathbf{U}^{(N)}$

$\underline{x}, \bar{x}$ : lower and upper bounds for the $x$ scalar

$\mathrm{Co}(\cdots)$ : convex hull (set of all convex comb.).

\section{Basic Concepts}

The section briefly discusses the related concepts of tensor algebra, polytopic LPV/qLPV modeling, and the goals of TP Model Transformation introducing the notations that are used in the followings.
3.1. Tensor Algebra. First, the key definitions and properties of tensor algebra of De Lathauwer et al. [18] are recalled and extended to Hilbert spaces by considering multidimensional arrays on a Hilbert space denoted by $H$ in general.

They can be multiplied with real matrices along the $n$th index that is called $n$-mode tensor product.

Definition 1 ( $n$-mode tensor product). The $n$-mode product of a tensor $\mathscr{A} \in H^{I_{1} \times \cdots \times I_{N}}$ and the matrix $\mathbf{U} \in \mathbb{R}^{L \times I_{n}}$, denoted by $\mathscr{A} \times{ }_{n} \mathrm{U}$, is a tensor with size $I_{1} \times \cdots \times I_{n-1} \times L \times I_{n+1} \times \cdots \times I_{N}$ that is given by

$$
\left(\mathscr{A} \times{ }_{n} \mathbf{U}\right)_{i_{1}, \ldots, i_{n-1}, l, i_{n+1}, \ldots, i_{N}}=\sum_{i_{n}} \mathfrak{a}_{i_{1}, \ldots, i_{N}} u_{l, i_{n}} .
$$

The definition implies the following properties.

Lemma 2 (commutativity of $n \neq l$-mode tensor products). Given the tensor $\mathscr{A} \in H^{I_{1} \times \cdots \times I_{N}}$ and the matrices $\mathbf{U} \in \mathbb{R}^{J \times I_{n}}$, $\mathbf{V} \in \mathbb{R}^{K \times I_{l}}(n \neq l)$, one has

$$
\left(\mathscr{A} \times{ }_{n} \mathbf{U}\right) \times{ }_{l} \mathbf{V}=\left(\mathscr{A} \times{ }_{l} \mathbf{V}\right) \times{ }_{n} \mathbf{U}
$$

Lemma 3 (multiple $n$-mode tensor products). Given the tensor $\mathscr{A} \in H^{I_{1} \times \cdots \times I_{N}}$ and the matrices $\mathbf{U} \in \mathbb{R}^{J \times I_{n}}, \mathbf{V} \in \mathbb{R}^{M \times J}$, one has

$$
\left(\mathscr{A} \times_{n} \mathbf{U}\right) \times_{n} \mathbf{V}=\mathscr{A} \times{ }_{n}(\mathbf{V U})
$$

The inner product and norm are defined.

Definition 4 (inner product and norm of tensors). The inner product $\langle\mathscr{A}, \mathscr{B}\rangle$ of tensors $\mathscr{A}, \mathscr{B} \in H^{I_{1} \times \cdots \times I_{N}}$ is defined as

$$
\langle\mathscr{A}, \mathscr{B}\rangle=\sum_{i_{1}} \cdots \sum_{i_{N}}\left\langle\mathfrak{a}_{i_{1}, \ldots, i_{N}}, \mathfrak{b}_{i_{1}, \ldots, i_{N}}\right\rangle .
$$

Then the Frobenius norm of a tensor $\mathscr{A}$ is defined as $\|\mathscr{A}\|=$ $\sqrt{\langle\mathscr{A}, \mathscr{A}\rangle}$.

To perform other matrix operations (e.g., SVD) along the $n$th index, the tensor can be unfolded to a matrix and restored back to tensor.

Definition 5 ( $n$-mode unfold tensor). Assume an $N$ th-order tensor $\mathscr{A} \in H^{I_{1} \times \cdots \times I_{N}}$, where the elements can be described on an orthonormal basis with finite $R$ elements; then its $n$ mode matrix unfolding is denoted by $\mathbf{A}_{(n)}$ with a size of $I_{n} \times$ $\left(I_{n+1} \cdots I_{N} R I_{1} \cdots I_{n-1}\right)$ and it contains the $r$ th coordinate of $\mathfrak{a}_{i_{1}, \ldots, i_{N}}$ element at the position $\left(i_{n}, j_{n}\right)$, where

$$
j_{n}=r+\sum_{l=1, l \neq n}^{N}\left(i_{l}-1\right) R \prod_{m=1, m \neq n}^{l-1} I_{m} .
$$

3.2. Hilbert-Space Valued Multivariate Functions. Consider the $\mathfrak{c}: X \rightarrow H$ function, where $X$ is a hyperrectangle on the real numbers $X=\left[\underline{x}_{1}, \bar{x}_{1}\right] \times \cdots \times\left[\underline{x}_{L}, \bar{x}_{L}\right] \subset \mathbb{R}^{L}$ and $H$ is a Hilbert space in general. The measure of $A \subseteq X$ set will be denoted as $V(A)=\int_{A} d x_{1} \cdots d x_{L}$. 
Definition 6 (inner product and norm). The inner product of $\mathfrak{b}, \mathfrak{c}: X \rightarrow H$ functions: we will use the following quantity:

$$
\langle\mathfrak{b}, \mathfrak{c}\rangle=\frac{1}{V(X)} \int_{\mathbf{x} \in X}\langle\mathfrak{b}(\mathbf{x}), \mathfrak{c}(\mathbf{x})\rangle V(d \mathbf{x})
$$

then their norms are as follows: $\|\mathfrak{c}\|=\sqrt{\langle\mathfrak{c}, \mathfrak{c}\rangle}$.

Along the paper, we will assume that for the considered functions this norm exists and it is finite without mentioning it.

The decomposition

$$
\mathfrak{c}(\mathbf{x})=\sum_{i=1}^{I} \mathfrak{c}_{i} f_{i}(\mathbf{x}) ;
$$

will be called

(i) orthonormal, if the weighting functions are orthonormal as $\left\langle f_{i}, f_{j}\right\rangle=\delta_{i j} \forall i, j=1 \cdots I$,

(ii) homogeneous, if $f_{I}(\mathbf{x})=1 \forall \mathbf{x} \in \mathbf{X}$,

(iii) polytopic, if the $f_{i}$ functions denote convex combinations as

$$
\sum_{i=1}^{I} f_{i}(\mathbf{x})=1, \quad f_{i}(\mathbf{x}) \geq 0 \forall i \forall \mathbf{x} \in X .
$$

Then, in geometric sense, the $\left\{\mathfrak{c}_{1}, \ldots, \mathfrak{c}_{I}\right\}$ vertices construct an enclosing polytope for the image of $\mathfrak{c}(\mathbf{x})$. Its elements are inside the polytope because they can be described as a convex combination of the vertices. In these cases, letter $w$ will denote the weighting functions through the paper.

3.3. Polytopic LPV/qLPV Modeling. Consider the following form of LPV/qLPV models:

$$
\begin{aligned}
& {\left[\begin{array}{c}
\dot{\mathbf{x}}(t) \\
\mathbf{y}(t) \\
\mathbf{z}(t)
\end{array}\right]} \\
& \quad=\left[\begin{array}{ccc}
\mathbf{A}(\mathbf{p}(t)) & \mathbf{B}_{u}(\mathbf{p}(t)) & \mathbf{B}_{w}(\mathbf{p}(t)) \\
\mathbf{C}_{y}(\mathbf{p}(t)) & \mathbf{D}_{y u}(\mathbf{p}(t)) & \mathbf{D}_{y w}(\mathbf{p}(t)) \\
\mathbf{C}_{z}(\mathbf{p}(t)) & \mathbf{D}_{z u}(\mathbf{p}(t)) & \mathbf{D}_{z w}(\mathbf{p}(t))
\end{array}\right]\left[\begin{array}{l}
\mathbf{x}(t) \\
\mathbf{u}(t) \\
\mathbf{w}(t)
\end{array}\right],
\end{aligned}
$$

where

(i) $\mathbf{x}(t)$ denotes the state variables, $\mathbf{u}(t)$ the control inputs, $\mathbf{w}(t)$ the disturbances, $\mathbf{y}(t)$ the measured outputs, and $\mathbf{z}(t)$ the performance outputs,

(ii) it is defined on a hyperrectangular parameter domain:

$$
\mathbf{p} \in \Omega=\left[\underline{p}_{1}, \bar{p}_{1}\right] \times \cdots \times\left[\underline{p}_{N}, \bar{p}_{N}\right] \subset \mathbb{R}^{N}
$$

(iii) for the sake of brevity, the parameter-dependent system matrices will be denoted as

$$
\mathbf{S}(\mathbf{p})=\left[\begin{array}{ccc}
\mathbf{A}(\mathbf{p}) & \mathbf{B}_{u}(\mathbf{p}) & \mathbf{B}_{w}(\mathbf{p}) \\
\mathbf{C}_{y}(\mathbf{p}) & \mathbf{D}_{y u}(\mathbf{p}) & \mathbf{D}_{y w}(\mathbf{p}) \\
\mathbf{C}_{z}(\mathbf{p}) & \mathbf{D}_{z u}(\mathbf{p}) & \mathbf{D}_{z w}(\mathbf{p})
\end{array}\right]
$$

so we have the $\Omega \rightarrow \mathbb{S}$ function, where $\mathbb{S}$ denotes the space of real matrices with appropriate size.

That is often extended with delayed inputs, delayed states, and so on according to the dynamics of the investigated system; see [19].

Polytopic models are polytopic decomposition of the $\mathbf{S}(\mathbf{p})$ system matrix. They are described as convex combinations of so-called vertex system matrices, as

$$
\mathbf{S}(\mathbf{p})=\sum_{r=1}^{R} \mathbf{S}_{r} h_{r}(\mathbf{p}), \quad \forall \mathbf{p} \in \Omega,
$$

and this form allows for using LMI-based control analysis and synthesis methods.

3.4. TP Model Transformation. TP Model Transformation is aimed at transforming the parameter-dependent system matrix $\mathbf{S}(\mathbf{p})$ into polytopic form with decoupled parameter dependencies, resulting in a nested parameter-wise polytopic representation that is expressed as multiple tensor products.

Definition 7 (polytopic TP Model). Polytopic TP Models are (q)LPV models with system matrices:

$$
\mathbf{S}(\mathbf{p})=\mathcal{S} \underset{n=1}{\stackrel{\otimes}{\otimes}} \mathbf{w}^{(n)}\left(p_{n}\right),
$$

in which

(i) the $\mathcal{S} \in \mathbb{S}^{J_{1} \times \cdots \times J_{N}}$ core tensor contains the $\mathbf{S}_{j_{1}, \ldots, j_{N}}$ vertex system matrices of the polytopic model,

(ii) the $w_{1}^{(n)}\left(p_{n}\right), \ldots, w_{J_{n}}^{(n)}\left(p_{n}\right) n$-mode weighting functions denote convex combinations $\forall p_{n} \in\left[\underline{p}_{n}, \bar{p}_{n}\right]$.

Let us recall its expanded form and highlight that it is polytopic for all parameter dependencies because the short TP notation can be extended as

$$
\begin{aligned}
\mathbf{S}(\mathbf{p}) & =\sum_{j_{1}=1}^{J_{1}} w_{j_{1}}^{(1)}\left(p_{1}\right) \sum_{j_{2}=1}^{J_{2}} \ldots \sum_{j_{N}=1}^{J_{N}} w_{j_{N}}^{(N)}\left(p_{N}\right) \mathbf{S}_{j_{1}, \ldots, j_{N}} \\
& =\sum_{j=1}^{J_{n}}\left(\mathcal{S}_{j_{n}=j} \underset{\substack{\bigotimes \\
l=1, l \neq n}}{N} \mathbf{w}^{(l)}\left(p_{l}\right)\right) w_{j}^{(n)}\left(p_{n}\right)
\end{aligned}
$$

for all $n=1 \cdots N$.

It is easy to see that this form is a special polytopic model. This way, the polytopic model-based control analysis and synthesis methodology can apply to them. Furthermore, the parameter separated structure can be exploited during control analysis and synthesis; for more details, see [5].

\section{Affine Decomposition of Univariate Functions}

The section shows the role of affine geometry in the derivation of polytopic decomposition of univariate functions, and it introduces the Affine Singular Value Decomposition to represent the geometric structure in a unique way that will be applied in the Affine TP Model. 
4.1. Enclosing Polytope on the Affine Hull. Consider the univariate $c:[\underline{x}, \bar{x}](\subset \mathbb{R}) \rightarrow H$ function, where $H$ is a Hilbert space. Denote its image to be enclosed by the polytopic form as

$$
\mathfrak{c}=\{\mathfrak{c}(x) \mid x \in[\underline{x}, \bar{x}]\} \subset H .
$$

Although the considered Hilbert space can be higher dimensional, there may exist polytopic descriptions with a finite number of vertices. It depends on the dimension of the so-called affine hull that is the minimum dimensional affine subspace which contains every object. It can be expressed as the set of affine combinations of the values of the function

$$
\mathfrak{A}=\left\{\int_{x=\underline{x}}^{\bar{x}} \alpha(x) \mathfrak{c}(x) d x \mid \int_{x=\underline{x}}^{\bar{x}} \alpha(x) d x=1\right\} \supset \mathfrak{S} .
$$

The dimension of the affine hull is called affine dimension and denoted by $D$. Then the elements of the $\mathfrak{r}$ image can be given as the sum of a value on the $\left(\mathfrak{a}_{1}, \ldots, \mathfrak{a}_{D}\right)$ basis and an $\left(\mathfrak{a}_{D+1}\right)$ offset, by applying homogeneous coordinates $\mathbf{v}(x)$ as

$$
\mathfrak{c}(x)=\sum_{d=1}^{D} u_{d}(x) \mathfrak{a}_{d}+\mathfrak{a}_{D+1}=\mathbf{v}(x)\left[\begin{array}{c}
\mathfrak{a}_{1} \\
\vdots \\
\mathfrak{a}_{D+1}
\end{array}\right]
$$

where $\mathbf{v}(x)=[\mathbf{u}(x) 1]=\left[u_{1}(x) \cdots u_{D}(x) 1\right]$. With this description, the objects are characterized by coordinates $\mathbf{u}(x)=\left[u_{1}(x), \ldots, u_{D}(x)\right]$ on the affine hull.

Obtaining an enclosing polytope for the $\mathbf{u}(x)$ coordinates in the $D$-dimensional Euclidean space with vertices $\left\{\mathbf{r}_{1}, \ldots, \mathbf{r}_{J}\right\}$ as

$$
\{\mathbf{u}(x) \mid x \in[\underline{x}, \bar{x}]\} \subseteq \operatorname{Co}\left(\mathbf{r}_{1}, \ldots, \mathbf{r}_{J}\right) \subset \mathbb{R}^{D},
$$

the $\mathbf{v}(x)$ homogeneous coordinates can be expressed as convex combinations of the vertices with weights $\left(w_{1}(x)\right.$, $\left.\ldots, w_{J}(x)\right)$ as

$$
\mathbf{v}(x)=\mathbf{w}(x) \mathbf{R}, \quad \text { where } \mathbf{R}=\left[\begin{array}{cc}
\mathbf{r}_{1} & 1 \\
\vdots & \vdots \\
\mathbf{r}_{J} & 1
\end{array}\right]
$$

and it provides an enclosing polytope for the $\mathfrak{E}$ image set with the following vertices:

$$
\mathfrak{S}_{j}=\left[\begin{array}{ll}
\mathbf{r}_{j} & 1
\end{array}\right]\left[\begin{array}{c}
\mathfrak{a}_{1} \\
\vdots \\
\mathfrak{a}_{D+1}
\end{array}\right] \quad(\forall j=1 \cdots J)
$$

because it can be described as their convex combinations:

$$
\begin{aligned}
\mathfrak{c}(x) & =\mathbf{v}(x)\left[\begin{array}{c}
\mathfrak{a}_{1} \\
\vdots \\
\mathfrak{a}_{D+1}
\end{array}\right]=\mathbf{w}(x) \mathbf{R}\left[\begin{array}{c}
\mathfrak{a}_{1} \\
\vdots \\
\mathfrak{a}_{D+1}
\end{array}\right] \\
& =\mathbf{w}(x)\left[\begin{array}{c}
\mathfrak{G}_{1} \\
\vdots \\
\mathfrak{g}_{J}
\end{array}\right] .
\end{aligned}
$$

This way, the polytopic description can be constructed for the original image in the $H$ space by considering the $D$ dimensional geometric problem.

4.2. Affine Singular Value Decomposition of Univariate Functions. Consider the description on the affine hull in (17) and restrict it to orthogonal $\left(\mathfrak{a}_{1}, \ldots, \mathfrak{a}_{D}\right)$ bases and homogeneous, orthonormal $\left(v_{1}(x), \ldots, v_{D+1}(x)\right)$ coordinate functions. Then we can define the following unique form that is called Affine Singular Value Decomposition.

Definition 8 (affine SVD (ASVD)). The form represented by (17) is called affine SVD of $c$ function if it is a homogeneous, orthonormal decomposition and the $\mathfrak{a}_{i} \in H(i=1 \cdots D)$ elements of the basis are orthogonal and ordered by their norms as

$$
\begin{aligned}
\left\langle\mathfrak{a}_{i}, \mathfrak{a}_{j}\right\rangle & =\delta_{i j} \sigma_{i}^{2} \quad \forall i, j=1 \cdots D, \\
\sigma_{1} & \geq \cdots \geq \sigma_{D}>0
\end{aligned}
$$

which are called singular values.

The decomposition's uniqueness property is inherited from the uniqueness of SVD.

Lemma 9 (uniqueness of ASVD). The $\sigma_{1}, \ldots, \sigma_{D}$ singular values and the $\mathfrak{a}_{D+1}$ offset are unique.

Now consider the ordered singular values and let $\left(m_{1}\right.$, $\left.m_{2}, \ldots\right)$ denote their multiplicities such that

$$
\begin{aligned}
\underbrace{\sigma_{1}=\cdots=\sigma_{m_{1}}}_{m_{1}} & >\underbrace{\sigma_{m_{1}+1}=\cdots=\sigma_{m_{1}+m_{2}}}_{m_{2}}>\cdots>\sigma_{D} \\
& >0 .
\end{aligned}
$$

Then the forms and only these forms are valid decomposition

$$
\mathfrak{c}(x)=\sum_{d=1}^{D+1} v_{d}^{\prime}(x) \mathfrak{a}_{d}^{\prime}
$$

where

$$
\begin{aligned}
{\left[v_{1}^{\prime}(x) \cdots v_{D}^{\prime}(x)\right] } & =\left[v_{1}(x) \cdots v_{D}(x)\right] \mathbf{T}, \\
{\left[\mathfrak{a}_{1}^{\prime} \cdots \mathfrak{a}_{D}^{\prime}\right]^{T} } & =\mathbf{T}^{T}\left[\mathfrak{a}_{1} \cdots \mathfrak{a}_{D}\right]^{T},
\end{aligned}
$$




$$
\begin{aligned}
v_{D+1}^{\prime}(x) & =v_{D+1}(x), \\
\mathfrak{a}_{D+1}^{\prime} & =\mathfrak{a}_{D+1}, \\
\mathbf{T} & =\operatorname{blockdiag}\left(\mathbf{Q}_{1}, \mathbf{Q}_{2}, \ldots\right),
\end{aligned}
$$

and $\mathbf{Q}_{i}$ are arbitrary real orthogonal matrices with size $m_{i} \times m_{i}$, respectively.

Proof. These kinds of decomposition are ASVD because

(i) by multiplying the orthonormal $v_{d}(x) d=1 \cdots D$ functions with a $\mathbf{T}$ orthogonal matrix, they remain orthonormal,

(ii) by multiplying the orthogonal $\mathfrak{a}_{d}$ values of the same norm with a $\mathbf{Q}_{i}$ orthogonal matrix, they maintain their orthogonality and norm as well. This way, the singular values and their order do not change.

Only this kind of decomposition is ASVD, because

(i) to ensure the $v_{D+1}^{\prime}(x)=1$ and the orthonormality of $v_{d}(x)$ functions, the offset part cannot change:

$$
\int_{x=\underline{x}}^{\bar{x}} \mathfrak{c}(x) \frac{d x}{\bar{x}-\underline{x}}=\sum_{d=1}^{D+1}\left\langle v_{d}(x), 1\right\rangle a_{d}=a_{D+1},
$$

(ii) the remaining part must be the SVD of function $\left(\mathfrak{c}(x)-\mathfrak{a}_{D+1}\right)$ inheriting its uniqueness properties, which results in the structure of $\mathbf{T}$.

Obviously, if every singular value is different, only the signs of $\mathfrak{a}_{d}$ objects and $v_{d}(x)$ functions $(d=1, \ldots, D)$ can be varied, because the lemma allows for only $\mathbf{Q}_{i}= \pm 1$ values in these cases.

Lemma 10 (complexity trade-off). Consider the affine SVD in (17) with $D$ singular values, where $D$ is the dimension of the affine hull.

The best $d<D$-dimensional approximation (in terms of the defined norm) can be obtained as

$$
\mathfrak{c}(x)=\sum_{l=1}^{d} v_{l}(x) \mathfrak{a}_{l}+\mathfrak{a}_{D+1} .
$$

Proof. It was shown in (26) that the average value of $\mathfrak{c}$ function is $\mathfrak{a}_{D+1}$ so it is the best $d=0$-dimensional approximation.

And if the best $d$-dimensional approximation is known, the best $d+1$-dimensional can be obtained by adding the a product with maximal possible norm (as in the EckhartYoung theorem [20]), which is here $\mathfrak{a}_{d+1} v_{d+1}(x)$.

Because the complexity of enclosing polytope generation depends on the dimension of the affine hull, this property allows for its reduction with minimal error in the defined norm.

The following lemma describes the numerical reconstruction assuming a vector function given as a homogeneous, orthonormal decomposition.
Lemma 11 (ASVD from a homogen. orthonorm. decomp.). Consider the $\mathbf{s}:[\underline{x}, \bar{x}] \rightarrow \mathbb{R}^{R}$ function, which is given as a homogeneous, orthonormal decomposition

$$
\mathbf{s}(x)=\sum_{m=1}^{M} f_{m}(x) \mathbf{k}_{m}
$$

in matrix form as $\mathbf{s}(x)=\mathbf{f}(x) \mathbf{K}$.

Then $\mathbf{s}(x)=\mathbf{v}(x) \mathbf{K}^{\prime}$ ASVD can be obtained as

$$
\begin{aligned}
\mathbf{v}(x) & =\mathbf{f}(x)\left[\begin{array}{ll}
\mathbf{U} & 0 \\
\mathbf{0} & 1
\end{array}\right], \\
\mathbf{K}^{\prime} & =\left[\begin{array}{l}
\mathbf{S V}^{T} \\
\mathbf{k}_{M}
\end{array}\right],
\end{aligned}
$$

where the matrices $\mathbf{U}, \mathbf{S}$, and $\mathbf{V}$ come from the SVD computation:

$$
\mathbf{U S V}^{T}=\operatorname{svd}\left(\left[\begin{array}{c}
\mathbf{k}_{1} \\
\vdots \\
\mathbf{k}_{M-1}
\end{array}\right]\right)
$$

omitting the zero singular values and the corresponding columns of singular matrices.

Proof. $\mathbf{v}(x)$ is orthonormal, because $\mathbf{f}(x)$ is orthonormal and blockdiag $(\mathbf{U}, 1)$ is orthogonal. It is homogeneous because $v_{D+1}(x)=f_{M}(x)=1$. The $\mathbf{k}_{d}^{\prime}$ values $(d=1 \cdots D)$ are orthogonal and ordered by norm from properties of SVD.

\section{Definition of Affine Tensor Product Form}

This section presents the derivation of polytopic TP forms for multivariate functions

$$
\mathfrak{c}: \Omega \longrightarrow H
$$

based on the Affine TP form, which represents the affine geometric structure for all parameter dependency, respectively.

Definition 12 (Affine TP form). The following form of function (31)

$$
\mathfrak{c}(\mathbf{p})=\mathscr{C}^{\mathrm{aff}} \underset{n=1}{\stackrel{\otimes}{N} \mathbf{v}^{(n)}}\left(p_{n}\right)
$$

is called Affine TP form, in which the $\mathscr{C}^{\text {aff }}$ core tensor is on $H$ as $\mathscr{C}^{\text {aff }} \in H^{\left(D_{1}+1\right) \times \cdots \times\left(D_{N}+1\right)}$, the $D_{n}(n=1 \cdots N)$ values are called $n$-mode dimensions, and the $n$-mode expansion of (32)

$$
\mathfrak{c}(\mathbf{p})=\sum_{d=1}^{D_{n}+1}\left(\mathscr{C}_{d_{n}=d}^{\mathrm{aff}} \underset{l=1, l \neq n}{\stackrel{N}{\otimes}} \mathbf{v}^{(l)}\left(p_{l}\right)\right) v_{d}^{(n)}\left(p_{n}\right)
$$

is an ASVD with $\sigma_{1}^{(n)}, \ldots, \sigma_{D_{n}}^{(n)}$ singular values for all $n$, respectively. 
Remark 13 (ASVD on functions). The definition exploits the fact that functions with norm in Definition 6 constitute Hilbert spaces. This way, the $\mathfrak{c}(\mathbf{p})$ function can be considered as a univariate function

$$
\left[\underline{p}_{n}, \bar{p}_{n}\right] \longrightarrow \mathbb{H}
$$

for all $n=1 \cdots N$, where $\mathbb{W}$ is the Hilbert space of functions

$$
\begin{aligned}
\Omega_{1} \times \cdots \times \Omega_{n-1} \times \Omega_{n+1} \times \cdots \times \Omega_{N} \longrightarrow H & \\
& \left(\Omega_{i}=\left[\underline{p}_{i}, \bar{p}_{i}\right]\right),
\end{aligned}
$$

and the ASVD is defined for it.

The polytopic TP form can be obtained by determining enclosing polytopes for all $\mathbf{v}^{(n)}\left(p_{n}\right)$ trajectories in the $D_{n^{-}}$ dimensional spaces for all $n=1 \cdots N$ and applying the following theorem.

Theorem 14 (derivation of polytopic TP form). If for all $n=$ $1 \cdots N$ the vertices $\mathbf{r}_{1}^{(n)}, \ldots, \mathbf{r}_{J_{n}}^{(n)}$ construct enclosing polytopes for trajectories $\mathbf{v}^{(n)}\left(p_{n}\right)$, they can be expressed as $\mathbf{v}^{(n)}\left(p_{n}\right)=$ $\mathbf{w}^{(n)}\left(p_{n}\right) \mathbf{R}^{(n)}$ (see (19)). Then

$$
\begin{aligned}
& \mathfrak{c}(\mathbf{p})=\mathscr{C}^{a f f} \underset{n=1}{\stackrel{\otimes}{\otimes}}\left(\mathbf{w}^{(n)}\left(p_{n}\right) \mathbf{R}^{(n)}\right) \\
& =\left(\mathscr{C}^{a f f} \underset{n=1}{\underset{\otimes}{\otimes}} \mathbf{R}^{(n)}\right) \underset{n=1}{\mathbb{\otimes}} \mathbf{w}^{(n)}\left(p_{n}\right)
\end{aligned}
$$

which is a polytopic TP form.

Proof. From Section 4.1, the uniqueness of the Affine TP form can be characterized by the following theorem.

Theorem 15 (uniqueness). The $\sigma_{d}^{(n)}$ singular values are unique; let $\left(m_{1}^{(n)}, m_{2}^{(n)}, \ldots\right)$ denote their multiplicities as in (23).

If (32) is an Affine TP form, the following and only the following forms are Affine TP Models:

$$
\mathfrak{c}(\mathbf{p})=\left(\mathscr{C}^{a f f} \underset{n=1}{\stackrel{\otimes}{\otimes}} \mathbf{T}^{(n) T}\right) \underset{n=1}{\stackrel{\otimes}{\otimes}}\left(\mathbf{v}^{(n)}\left(p_{n}\right) \mathbf{T}^{(n)}\right),
$$

where the matrices are defined as $\mathbf{T}^{(n)}=\operatorname{diag}\left(\mathbf{T}_{0}^{(n)}, 1\right)$ and $\mathbf{T}_{0}^{(n)}$ is a block-diagonal matrix constructed by arbitrary orthogonal matrices with sizes $m_{1}^{(n)} \times m_{1}^{(n)}, m_{2}^{(n)} \times m_{2}^{(n)}$, and so on as shown in Lemma 9.

Proof. Only these forms are allowed by uniqueness properties of ASVD (see Lemma 9) and their $n$-mode expansions

$$
\begin{aligned}
\mathfrak{c}(\mathbf{p})= & \sum_{d=1}^{D_{n}+1}\left(\mathscr{C}^{\mathrm{aff}} \underset{\substack{\mathbb{\otimes} \\
\mathrm{\otimes}}, l \neq n}{N} \mathbf{v}^{(l)}\left(p_{l}\right) \times_{n} \mathbf{T}^{(n) T}\right)_{d_{n}=d} \\
& \cdot\left(\mathbf{v}^{(n)}\left(p_{n}\right) \mathbf{T}^{(n)}\right)_{d}
\end{aligned}
$$

show that these forms are ASVD, so the TP form is affine.
The form enables the $n$-mode dimension reductions with the following error (regarding the defined norm) based on the properties of TP forms on orthonormal weighting functions, which are discussed in the Appendix.

Theorem 16 (complexity reduction). The reduction of one $n$ mode dimension from $D_{n}$ to $D_{n}^{\prime}<D_{n}$ with minimal error in the defined norm can be achieved by omitting the $\left(D_{n}^{\prime}+\right.$ $1), \ldots, D_{n}$ th subtensors of $\mathscr{C}^{\text {aff }}$ and the corresponding elements of $\mathbf{v}^{(n)}\left(p_{n}\right)$. Then the error is

$$
\|\mathfrak{c}-\widehat{\mathfrak{c}}\|^{2}=\sum_{d=D_{n}^{\prime}+1}^{D_{n}} \sigma_{d}^{(n) 2} .
$$

The approximation error of dimension reduction in multiple $(n=1 \cdots N)$ parameter dependencies is bounded as

$$
\|\mathfrak{c}-\widehat{\mathfrak{c}}\|^{2} \leq \sum_{n=1}^{N} \sum_{d=D_{n}^{\prime}+1}^{D_{n}} \sigma_{d}^{(n) 2} .
$$

Proof. Construct a tensor $\widehat{\mathscr{C}}^{\text {aff }}$ with the same sizes as $\mathscr{C}^{\text {aff }}$ that contains zeros in the omitted subtensors. Then, if $\Delta \mathscr{C}^{\text {aff }}=$ $\mathscr{C}^{\text {aff }}-\widehat{\mathscr{C}}^{\text {aff }}$, the approximation error can be written as

$$
\mathfrak{c}(\mathbf{p})-\widehat{\mathfrak{c}}(\mathbf{p})=\Delta \mathscr{C}^{\mathrm{aff}} \underset{n=1}{\mathbb{\bigotimes}} \mathbf{v}^{(n)}\left(p_{n}\right) .
$$

If only one $n$-mode dimension is decreased, the error of the approximation can be written as (based on Lemma A.2)

$$
\|\mathfrak{c}-\widehat{\mathfrak{c}}\|^{2}=\sum_{d=1}^{D_{n}+1}\left\|\Delta \mathscr{C}_{d_{n}=d}^{\mathrm{aff}}\right\|^{2}=\sum_{d=D_{n}^{\prime}+1}^{D_{n}} \sigma_{d}^{(n) 2}
$$

that is minimal as Lemma 10 indicated.

Considering the case when more than one n-mode dimension is decreased, the worst case (equality) of (40) occurs if there are zero elements in the intersection of the omitted subtensors. Otherwise, the error of the approximation is smaller.

Finally, the method is presented for its exact derivation or at least approximate reconstruction.

Method 17 (numerical reconstruction of Affine TP form). The first step is to obtain an initial TP form with the desired parameter groups

$$
\begin{aligned}
\widehat{\mathfrak{c}}(\mathbf{p}) & =\mathscr{D} \underset{n=1}{\mathbb{\otimes}} \boldsymbol{\alpha}^{(n)}\left(p_{n}\right) \\
& =\sum_{m_{1}=1}^{M_{1}} \cdots \sum_{m_{N}=1}^{M_{N}} \mathfrak{d}_{m_{1}, \ldots, m_{N}} \prod_{n=1}^{N} \alpha_{m_{n}}^{(n)}\left(p_{n}\right) .
\end{aligned}
$$

Here we describe two approaches for it.

Step $1 a$ (analytical initial form). If the function is analytically given, the $\mathfrak{c}(\mathbf{p})=\widehat{\mathfrak{c}}(\mathbf{p})$ initial form may be constructed analytically.

Step $1 b$ (discretization based initial form). The function can be approximated by the TP form as $\mathfrak{c}(\mathbf{p}) \approx \widehat{\mathfrak{c}}(\mathbf{p})$ via 
discretization in general: For each $\mathbf{p}_{n}$ parameter, choose $M_{n}$ discrete points denoted as $\left\{\ldots, \mathbf{g}_{m_{n}}^{(n)}, \ldots\right\}$ and appropriate $\boldsymbol{\alpha}^{(n)}\left(\mathbf{p}_{n}\right)$ interpolatory functions (as Lagrange polynomials, piecewise linear/constant functions, etc.).

Then the initial TP form (43) can be constructed to approximate the function by choosing elements of the core tensor $\mathfrak{D} \in H^{M_{1} \times M_{2} \times \cdots \times M_{N}}$ denoted by $\mathfrak{d}_{m_{1}, \ldots, m_{N}}$ which is the value of $\mathfrak{c}(\mathbf{p})$ function at $\left(\mathbf{g}_{m_{1}}^{(1)}, \mathbf{g}_{m_{2}}^{(2)}, \ldots\right)$.

Step 2 (homogeneous orthonormalization). Determine the homogeneous, orthonormal weighting functions $\gamma^{(n)}$ : [ $\underline{p}_{n}$, $\left.\bar{p}_{n}\right] \rightarrow \mathbb{R}^{L_{n}}$ as $\boldsymbol{\gamma}^{(n)}\left(p_{n}\right) \mathbf{T}^{(n)}=\boldsymbol{\alpha}^{(n)}\left(p_{n}\right)$ to obtain the following orthonormal TP form:

$$
\widehat{\mathfrak{c}}(\mathbf{p})=\mathscr{F} \underset{n=1}{\otimes} \gamma^{(n)}\left(p_{n}\right),
$$

where

$$
\mathscr{F}=\mathscr{D} \underset{n=1}{\mathbb{\otimes}} \mathbf{T}^{(n)}
$$

Some examples are Gram-Schmidt orthogonalization [21], the Householder transformation [22-24], or the Givens rotation [25].

Step 3 (sequential ASVD). Denote the TP form as

$$
\widehat{\mathfrak{c}}(\mathbf{p})=\mathscr{K} \underset{n=1}{\mathbb{\otimes}} \mathbf{f}^{(n)}\left(p_{n}\right),
$$

whose initial value is $\mathscr{K}=\mathscr{F}$ and $\mathbf{f}^{(n)}\left(p_{n}\right)=\gamma^{(n)}\left(p_{n}\right)$ for $n=$ $1 \cdots N$. form as

Then for index $n=1$, compute the ASVD of $\mathbf{f}^{(n)}\left(p_{n}\right) \mathbf{K}_{(n)}$

$$
\mathbf{f}^{(n)}\left(p_{n}\right) \mathbf{K}_{(n)}=\mathbf{v}^{(n)}\left(p_{n}\right) \mathbf{K}^{\prime}
$$

(see Lemma 11) and continue with $\mathscr{K}:=$ inv_unfold $\left(\mathbf{K}^{\prime}\right)$, $\mathbf{f}^{(n)}\left(p_{n}\right):=\mathbf{v}^{(n)}\left(p_{n}\right)$, and $n=n+1$ until $n \leq N$.

Then the resulting TP form is affine.

Proof. For TP forms on orthonormal weighting functions, if $\mathscr{K} \times{ }_{n} \mathbf{v}^{(n)}\left(p_{n}\right)$ is ASVD, then

$$
\left(\mathscr{K} \underset{l=1, l \neq n}{\mathbb{\otimes}} \mathbf{f}^{(l)}\left(p_{l}\right)\right) \times_{n} \mathbf{v}^{(n)}\left(p_{n}\right)
$$

is ASVD as well; see Lemma A.3 of the Appendix.

The method proves the existence of Affine TP forms for cases where the separation of parameter dependencies is possible, and it extends the previous approach by allowing exact analytical separation or the application of discretization with varying density along the parameter domain $\Omega$ with different interpolation strategies.

Remark 18. The sequential truncation approach (see [26]) can also be applied by using the complexity reductions in iterations of Step 3 in order to decrease the computational cost.
Remark 19. By applying SVD instead of ASVD in Step 3 (and optionally simple orthonormalization in Step 2), the method can be used to determine the so-called HOSVD-based TP form as well.

\section{Application for LPV/qLPV Models}

The results of the previous section are appropriate for system matrices $\mathbf{S}(\mathbf{p})$ of (q)LPV models (9). By defining the inner product and norm for $\mathbf{F}, \mathbf{G} \in \mathbb{S}$ system matrices as

$$
\langle\mathbf{F}, \mathbf{G}\rangle=\operatorname{trace}\left(\mathbf{F} \cdot \mathbf{G}^{T}\right), \quad\|\mathbf{F}\|=\sqrt{\langle\mathbf{F}, \mathbf{F}\rangle},
$$

the space $\mathbb{S}$ constitutes a Hilbert space and the following TP Model can be defined.

Definition 20 (Affine TP Model). The system matrix of the (q)LPV model (9) is given in Affine TP form as

$$
\mathbf{S}(\mathbf{p})=\mathcal{S}^{\mathrm{aff}} \underset{n=1}{\mathbb{N}} \mathbf{v}^{(n)}\left(p_{n}\right) ;
$$

see Definition 12.

The elements of core tensor $\mathcal{S}^{\text {aff }}$ are system matrices and the functions $\mathbf{v}^{(n)}\left(p_{n}\right)$ are $D_{n}$-dimensional trajectories given by homogeneous coordinates.

The uniqueness of the description is inherited from Theorem 15. Complexity (dimension of the affine hull) reduction can be done based on Theorem 16 but it must be mentioned that it does not give guarantee about its distribution along the parameter domain in terms of dynamical effects, and thus, it is not closely related to its dynamical properties in ill-conditioned cases. It means that if the omitted details are not only numerical error (representing essential information about the system dynamics), it is recommended to apply robust design methods taking into account the neglected part as in [27].

Furthermore, it has direct link with polytopic model generation based on Theorem 14 .

Corollary 21 (polytopic model generation). The determination of vertices $\mathbf{r}_{1}^{(n)}, \mathbf{r}_{2}^{(n)}, \ldots, \mathbf{r}_{J_{n}}^{(n)} \in \mathbb{R}^{D_{n}}\left(J_{n} \geq D_{n}+1\right)$ for all $n=1 \cdots N$ constructs an enclosing polytope for the $\left[v_{1}^{(n)}\left(p_{n}\right) \cdots v_{D_{n}}^{(n)}\left(p_{n}\right)\right]$ trajectory and the weighting functions $\mathbf{w}^{(n)}\left(p_{n}\right)$ (interpreting convex combination for all $p_{n}$ ) in such a way that

$$
\mathbf{w}^{(n)}\left(p_{n}\right) \mathbf{R}^{(n)}=\mathbf{v}^{(n)}\left(p_{n}\right)
$$

(as in (19)).

Then the polytopic TP Model (13) can be formalized with $\mathbf{w}^{(n)}\left(p_{n}\right)$ weighting functions and core tensor

$$
\mathcal{S}=\mathcal{S}^{a f f} \underset{n=1}{\stackrel{\otimes}{\otimes}} \mathbf{R}^{(n)} .
$$

There exist numerical methods for enclosing simplex polytope generation (where $J_{n}=D_{n}+1$ ) such as the Minimal 
Volume Simplex Approach [13] and other simplex methods: CNO, IRNO, and SNNN [15, 17]. The classical convex hull methods $[28,29]$ can also be applied, but they usually result in enclosing polytopes with too many vertices (up to infinity).

Fine-tuning manipulation/optimization is an important technique in polytopic model-based design. Similarly to the polytope generation methods, manipulation techniques are also immediately connectible to the Affine TP Model.

Corollary 22 (polytopic model manipulation). As manipulation of $\mathfrak{N} \subset\{1, \ldots, N\}$ mode enclosing polytopes, determinate the vertices $\mathbf{r}_{1}^{\prime(n)}, \mathbf{r}_{2}^{\prime(n)}, \ldots, \mathbf{r}_{J_{n}^{\prime}}^{\prime(n)} \in \mathbb{R}^{D_{n}}\left(J_{n}^{\prime} \geq D_{n}+1\right)$ and weighting functions $\mathbf{w}^{\prime(n)}\left(p_{n}\right)$ for all $n \in \mathfrak{N}$ that constructs an enclosing polytope for the trajectory $\left[v_{1}^{(n)}\left(p_{n}\right) \cdots v_{D_{n}}^{(n)}\left(p_{n}\right)\right]$ in such a way that $\mathbf{w}^{\prime(n)}\left(p_{n}\right) \mathbf{R}^{\prime(n)}=\mathbf{v}^{(n)}\left(p_{n}\right)$ as in (19), taking into account the control design experience with previous enclosing polytopes. ized as

Then the manipulated polytopic TP Model can be formal-

$$
\mathbf{S}(\mathbf{p})=\mathcal{S}^{\operatorname{man}} \underset{n=1, n \notin \mathfrak{N}}{\underset{\otimes}{\mathbb{N}}} \mathbf{w}^{(n)}\left(p_{n}\right) \underset{n \in \mathfrak{N}}{\mathbb{\otimes}} \mathbf{w}^{\prime(n)}\left(p_{n}\right),
$$

where

$$
\mathcal{S}^{\text {man }}=\mathcal{S}^{a f f} \underset{n=1, n \notin \mathfrak{M}}{\underset{\otimes}{\otimes}} \mathbf{R}^{(n)} \underset{n \in \mathfrak{M}}{\otimes} \mathbf{R}^{\prime(n)} .
$$

Relevant examples are the manipulation of the constraints in MVS method based on the achievable performance with the previous polytopes (see $[13,30]$ ) or the nonsimplex method where problematic regions are cut off from the polytope [16].

\section{Numerical Example}

This section discusses a control-related example that gives hands-on insight into a realistic design scenario.

Consider the translational oscillator with an eccentric rotational mass actuator (TORA) system shown in Figure 1. The goal of the control effort is to stabilize its translational motion using a rotational actuator [31-35].

The equation of motion is usually reformulated in the following dimensionless form:

$$
\begin{aligned}
& \chi^{\prime \prime}=-\chi+\epsilon \sin \theta, \\
& \theta^{\prime \prime}=\frac{\epsilon \chi \cos \theta+u}{1-\epsilon^{2} \cos ^{2} \theta}-\epsilon^{2} \frac{\left(\theta^{\prime 2}+1\right) \cos \theta}{1-\epsilon^{2} \cos ^{2} \theta} \sin \theta,
\end{aligned}
$$

where

$$
\begin{aligned}
\chi & =\xi+\epsilon \sin \theta, \\
\xi & =\sqrt{\frac{M+m}{I+m e^{2}}} q, \\
u & =\frac{M+m}{k\left(I+m e^{2}\right)} N,
\end{aligned}
$$

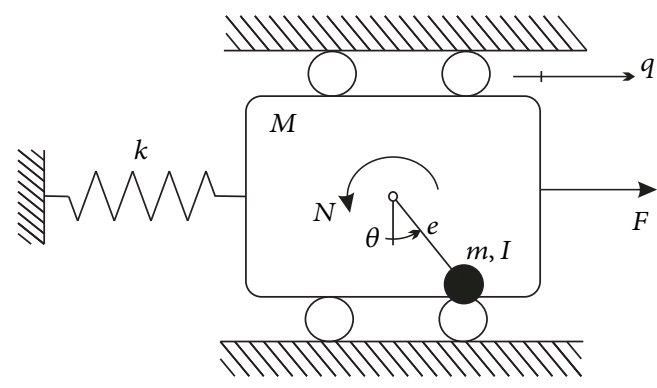

FIgURE 1: The mechanical model of the TORA system.

$$
\begin{aligned}
& \tau=\sqrt{\frac{k}{m+M} t}, \\
& \epsilon=\frac{m e}{\sqrt{\left(I+m e^{2}\right)(M+m)}},
\end{aligned}
$$

where $\xi$ denotes the dimensionless translational position, $u$ the dimensionless input, $\tau$ dimensionless time, and $\epsilon$ the coupling parameter.

The nonlinear ODE is used for the purpose of constructing the qLPV model; the state variables are chosen as

$$
\mathbf{x}=\left[\begin{array}{llll}
\chi & \chi^{\prime} & \theta & \theta^{\prime}
\end{array}\right]^{T},
$$

and the parameters as

$$
\begin{aligned}
& p_{1}=|\theta|, \\
& p_{2}=\frac{\left(\theta^{\prime 2}+1\right) \cos \theta}{1-\epsilon^{2} \cos ^{2} \theta} .
\end{aligned}
$$

Then the constructed qLPV realization reads

$$
\mathbf{x}^{\prime}=\left[\begin{array}{ll}
\mathbf{A}(\mathbf{p}) & \mathbf{B}(\mathbf{p})
\end{array}\right]\left[\begin{array}{l}
\mathbf{x} \\
u
\end{array}\right]=\mathbf{S}(\mathbf{p})\left[\begin{array}{l}
\mathbf{x} \\
u
\end{array}\right],
$$

where

$$
\mathbf{A}(\mathbf{p})=\left[\begin{array}{cccc}
0 & 1 & 0 & 0 \\
-1 & 0 & \epsilon \frac{\sin p_{1}}{p_{1}} & 0 \\
0 & 0 & 0 & 1 \\
\frac{\epsilon \cos p_{1}}{G\left(p_{1}\right)} & 0 & -\epsilon^{2} p_{2} \frac{\sin p_{1}}{p_{1}} & 0
\end{array}\right],
$$

$$
\mathbf{B}(\mathbf{p})=\left[\begin{array}{c}
0 \\
0 \\
0 \\
1 \\
G\left(p_{1}\right)
\end{array}\right],
$$



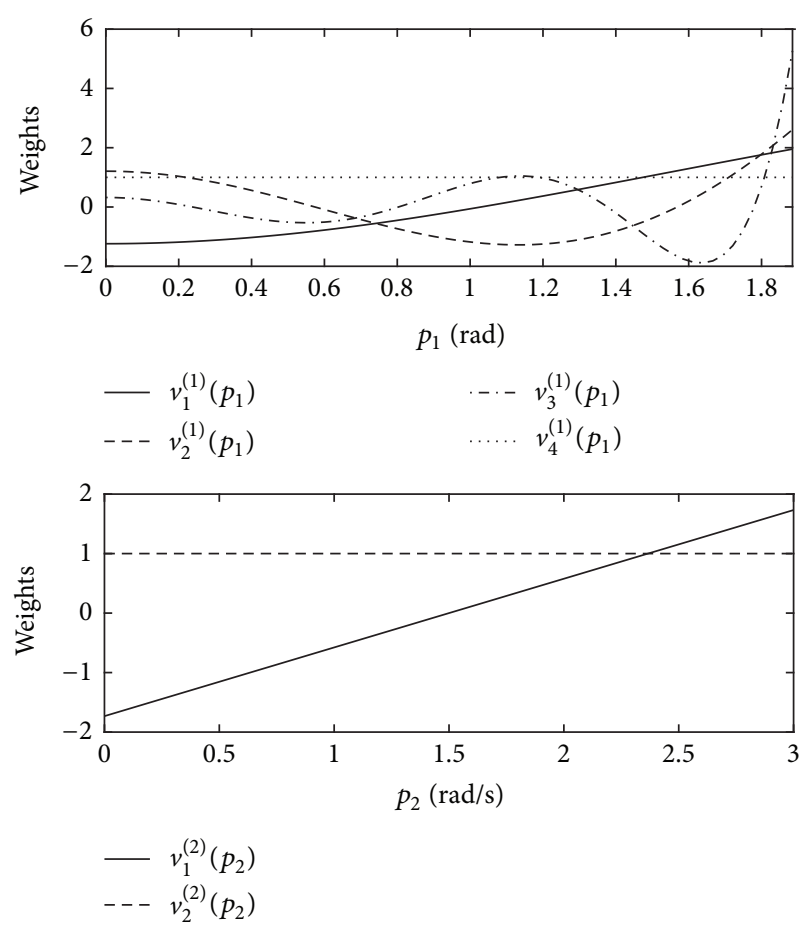

FIgURE 2: The $\mathbf{v}^{(1)}\left(p_{1}\right)$ and $\mathbf{v}^{(2)}\left(p_{2}\right)$ weighting functions in the Affine TP Model.

and here the $|\theta|<0.6 \pi|\dot{\theta}|<3(\mathrm{rad} / \mathrm{s})$ domain is considered with $\epsilon=0.2$ value.

In order to obtain the Affine TP form of function (59), the parameter dependencies are separated leading to an initial TP form with the following weighting functions:

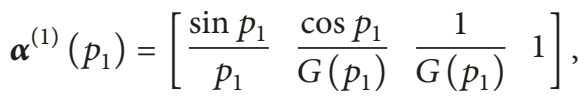

$$
\begin{aligned}
& \boldsymbol{\alpha}^{(2)}\left(p_{2}\right)=\left[\begin{array}{ll}
p_{2} & 1
\end{array}\right]
\end{aligned}
$$

performing Step 1a of Method 17. After orthogonalization and sequential ASVD, we get the affine form

$$
\begin{aligned}
\mathbf{S}(\mathbf{p}) & =\mathcal{S}^{\mathrm{aff}} \times_{1} \mathbf{v}^{(1)}\left(p_{1}\right) \times{ }_{2} \mathbf{v}^{(2)}\left(p_{2}\right) \\
& =\mathcal{S}^{\mathrm{aff}} \underset{n=1}{\stackrel{\bigotimes}{\otimes}} \mathbf{v}^{(n)}\left(p_{n}\right)
\end{aligned}
$$

where the $n$-mode dimensions are $D_{1}=3$ and $D_{2}=1$ and the corresponding weighting functions are depicted in Figure 2. The singular values: $\sigma_{1}^{(1)}=9.15 \cdot 10^{-2}, \sigma_{2}^{(1)}=$ $5.75 \cdot 10^{-3}, \sigma_{3}^{(1)}=1.91 \cdot 10^{-5}$, and $\sigma_{1}^{(2)}=2.90$. $10^{-2}$. (By applying discretization and interpolator functions, numerically reconstructed approximations of this TP form can be obtained.)

The enclosing polytope generation for $p_{2}$ dependency is trivial, because it is a one-dimensional problem. The vertices: $\mathbf{r}_{1}^{(2)}=\left[\begin{array}{ll}-1.731 & 1\end{array}\right]$ and $\mathbf{r}_{2}^{(2)}=\left[\begin{array}{ll}1.731 & 1\end{array}\right]$.

The three-dimensional problem of $p_{1}$ dependency is more challenging. The methods for generation of the enclosing polytope can be applied as MVS (or SNNN, CNO); see

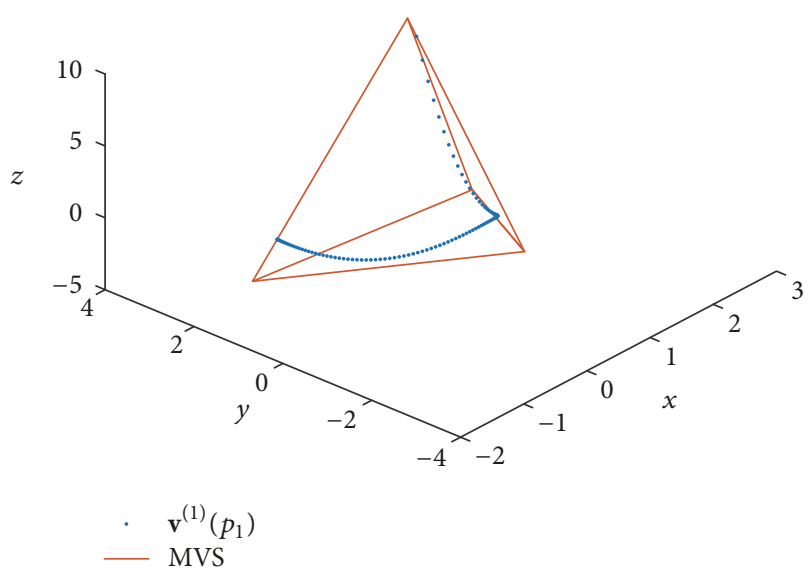

FIGURE 3: The MVS enclosing polytope for $\mathbf{v}^{(1)}\left(p_{1}\right)$ on the affine hull.
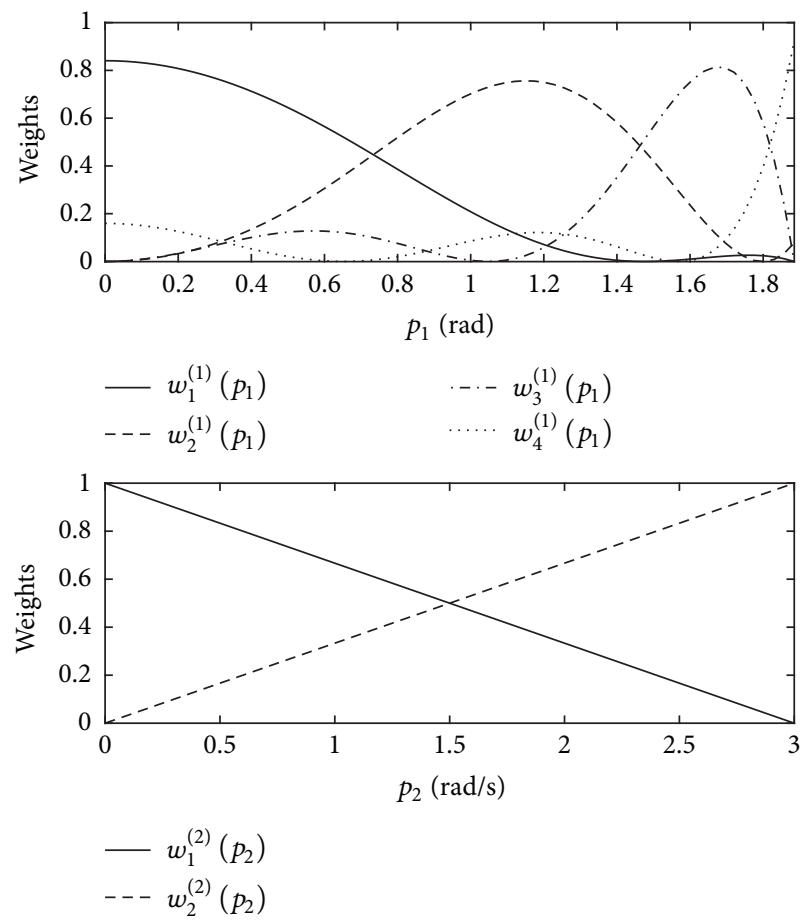

FIGURE 4: The $\mathbf{w}^{(1)}\left(p_{1}\right)$ and $\mathbf{w}^{(2)}\left(p_{2}\right)$ weighting functions in the polytopic TP Model.

Section 6. Figure 3 shows the resulting tetrahedron enclosing polytope and Figure 4 the weighting functions (denoting convex combinations) and the vertices are

$$
\begin{aligned}
& \mathbf{r}_{1}^{(1)}=\left[\begin{array}{llll}
-1.873 & 0.864 & -0.691 & 1
\end{array}\right], \\
& \mathbf{r}_{2}^{(1)}=\left[\begin{array}{llll}
0.201 & -2.311 & 0.661 & 1
\end{array}\right], \\
& \mathbf{r}_{3}^{(1)}=\left[\begin{array}{llll}
1.676 & 0.964 & -2.647 & 1
\end{array}\right], \\
& \mathbf{r}_{4}^{(1)}=\left[\begin{array}{llll}
2.102 & 3.027 & 5.659 & 1
\end{array}\right] .
\end{aligned}
$$


The resulting polytopic TP Model has two parameter dependencies, and it has the following general form:

$$
\mathbf{x}^{\prime}=\mathbf{S}(\mathbf{p})\left[\begin{array}{l}
\mathbf{x} \\
u
\end{array}\right],
$$

where

$$
\begin{aligned}
\mathbf{S}(\mathbf{p}) & =\mathcal{S} \underset{n=1}{\stackrel{2}{\otimes}} \mathbf{w}^{(n)}\left(p_{n}\right) \\
& =\sum_{j_{1}=1}^{2} \sum_{j_{2}=1}^{4} w_{j_{1}}^{(1)}\left(p_{1}\right) w_{j_{2}}^{(2)}\left(p_{2}\right)\left[\begin{array}{ll}
\mathbf{A}_{j_{1}, j_{2}} & \mathbf{B}_{j_{1}, j_{2}}
\end{array}\right] .
\end{aligned}
$$

For the sake of brevity, only the quadratic stabilization via state feedback problem is recalled and applied. To exploit the separated parameter dependencies, the controller-candidate depends only on the first parameter as

$$
u=\mathbf{F}\left(p_{1}\right) \mathbf{x}, \quad \mathbf{F}\left(p_{1}\right)=\sum_{j=1}^{4} w_{j}^{(1)}\left(p_{1}\right) \mathbf{F}_{j} .
$$

Then the closed-loop system is stable if there exist $\mathbf{X} \in \mathbb{R}^{4 \times 4}$ symmetric matrix and $\mathbf{M}_{j} \in \mathbb{R}^{1 \times 4} j=1 \cdots 4$ matrices such that

$$
\begin{aligned}
\mathbf{X} & >0, \\
\Gamma_{j_{1}, j_{1}, j_{2}}<0 \quad & \forall j_{1}=1 \cdots 4, j_{2}=1,2, \\
\Gamma_{j_{1}, i_{1}, j_{2}}<0 \quad \forall j_{1}=1 \cdots 4,1 \geq i_{1}<j_{1}, j_{2}=1,2, & \\
& \text { where } \Gamma_{j_{1}, i_{1}, j_{2}}=\operatorname{He}\left(\mathbf{A}_{j_{1}, j_{2}} \mathbf{X}+\mathbf{B}_{j_{1}, j_{2}} \mathbf{M}_{i_{1}}\right)
\end{aligned}
$$

and the gains of the state feedback are $\mathbf{F}_{j}=\mathbf{M}_{j} \mathbf{X}^{-1}$.

Here the problem is feasible with gains:

$$
\begin{aligned}
& \mathbf{F}_{1}=\left[\begin{array}{llll}
0.2419 & -0.5642 & -1.8000 & -0.6950
\end{array}\right], \\
& \mathbf{F}_{2}=\left[\begin{array}{llll}
0.4320 & -0.6009 & -1.9145 & -0.7264
\end{array}\right], \\
& \mathbf{F}_{3}=\left[\begin{array}{llll}
0.5588 & -0.6120 & -1.9458 & -0.7305
\end{array}\right], \\
& \mathbf{F}_{4}=\left[\begin{array}{llll}
0.5935 & -0.6124 & -1.9449 & -0.7285
\end{array}\right] .
\end{aligned}
$$

For more complex examples that apply other polytopic model generation, manipulation methods, and controller design techniques, see papers $[9,11,13,16,30]$.

\section{Conclusion}

The proposed Affine TP Model Transformation is a significant development in polytopic model-based control providing a general yet practically advantageous methodology for polytopic model generation. The unique Affine TP Model as a central concept serves as starting point for complexity reduction, polytopic model creation, and various polytope manipulation/optimization approaches helping to fully exploit the directly applicable powerful LMI-based synthesis methods. The most important benefits of the proposed intermediate TP form are the geometrically appropriate representation of the LPV structure in each dimension and the capability of dimension reduction with minimal error and low computational cost. In addition to the theoretical discussion, for the sake of technical completeness, an illustrative numerical example was provided to clearly show the practical merit of the Affine TP form.

In some sense, the paper sums up and consolidates the theoretical basis of TP Model Transformation that has been evolved in the past decade through the contribution of a broader research community.

\section{Appendix}

\section{Mathematical Background of Affine TP Form}

First, the following lemma highlights important properties of orthonormal decomposition.

Lemma A.1 (inner product and norm of orthonormal decomp.). If $\mathfrak{b}, \mathfrak{c}: X \rightarrow H$ functions are given with the same $\left\{\ldots, f_{i}(\mathbf{x}), \ldots\right\}$ orthonormal weighting functions, their inner product and norm can be computed as

$$
\langle\mathfrak{b}, \mathfrak{c}\rangle=\sum_{i=1}^{I}\left\langle\mathfrak{b}_{i}, \mathfrak{c}_{i}\right\rangle, \quad\|\mathfrak{c}\|=\sqrt{\sum_{i=1}^{I}\left\|\mathfrak{c}_{d}\right\|^{2}}
$$

This property appears in orthonormal TP forms in the following way.

Lemma A.2 (inner product and norm of orthonormal TP forms). If there are two TP functions given on the same orthonormal weighting function system as

$$
\begin{aligned}
& \mathfrak{c}(\mathbf{p})=\mathscr{C} \underset{n=1}{\stackrel{\otimes}{\otimes}} \mathbf{f}^{(n)}\left(p_{n}\right), \\
& \mathfrak{D}(\mathbf{p})=\mathscr{D} \underset{n=1}{\stackrel{\otimes}{\otimes}} \mathbf{f}^{(n)}\left(p_{n}\right),
\end{aligned}
$$

their inner product can be obtained as

$$
\langle\mathfrak{c}, \mathfrak{D}\rangle=\langle\mathscr{C}, \mathscr{D}\rangle .
$$

Furthermore, their norm can be derived as

$$
\|\mathfrak{c}\|=\|\mathscr{C}\|
$$

This way, the functions' orthogonality depends only on the orthogonality of the core tensors. Based on this property, the following lemma formalizes an important property of the Affine TP form.

Lemma A.3 (n-mode ASVD of orthonormal TP forms). If the weighting functions of TP form

$$
\mathfrak{f}(\mathbf{p})=\mathscr{K} \stackrel{\otimes}{\otimes}_{n=1}^{N} \mathbf{f}^{(n)}\left(p_{n}\right)
$$


are orthonormal, the following statements are equivalent for all $n=1 \cdots N$ :

(i) The following form is an ASVD along $p_{n}$ parameter

$$
\left(\mathscr{K} \underset{l=1, l \neq n}{\mathbb{N}} \mathbf{f}^{(l)}\left(p_{l}\right)\right) \times_{n} \mathbf{f}^{(n)}\left(p_{n}\right) .
$$

(ii) The form is an ASVD along $p_{n}$ parameter

$$
\mathscr{K} \times{ }_{n} \mathbf{f}^{(n)}\left(p_{n}\right) .
$$

Furthermore, their singular values are equal.

Proof. The requirements for the $\mathbf{f}^{(n)}\left(p_{n}\right)$ weighting functions are the same and the inner products of the bases are also equal because from Lemma A.2

$$
\begin{aligned}
& \left\langle\mathscr{K}_{d_{n}=i} \underset{l=1, l \neq n}{\mathbb{\otimes}} \mathbf{f}^{(l)}\left(p_{l}\right), \mathscr{K}_{d_{n}=j} \underset{l=1, l \neq n}{\mathbb{\otimes}} \mathbf{f}^{(l)}\left(p_{l}\right)\right\rangle \\
& \quad=\left\langle\mathscr{K}_{d_{n}=i}, \mathscr{K}_{d_{n}=j}\right\rangle ;
\end{aligned}
$$

this way, their orthogonality, order, and norms are the same.

Conclusively, the $n$-mode singular values can be obtained as norm of the $n$-mode subtensors of the core tensor and the ASVD on $p_{n}$ parameter dependency is invariant for inner transformations among orthonormal decomposition on other $p_{n}$ parameter dependencies.

\section{Conflicts of Interest}

The authors declare that they have no conflicts of interest.

\section{Acknowledgments}

The authors thankfully acknowledge the financial support of this work by the ÚNKP-16-3 and ÚNKP-16-4 New National Excellence Program of the Ministry of Human Capacities and the support of the Doctoral School of Applied Informatics and Applied Mathematics of Óbuda University and Research and Innovation Center of Óbuda University.

\section{References}

[1] S. Boyd, L. El Ghaoui, E . Feron, and V. Balakrishnan, Linear Matrix Inequalities in System and Control Theory, SIAM, Philadelphia, Pa, USA, 1994.

[2] P. Baranyi, "TP model transformation as a way to LMI-based controller design," IEEE Transactions on Industrial Electronics, vol. 51, no. 2, pp. 387-400, 2004.

[3] K. Tanaka and H. O. Wang, "Fuzzy control systems design and analysis a linear matrix inequality approach," in $1 \mathrm{em}$ plus $0.5 \mathrm{em}$ minus 0.4em, Wiley, New York, 2001.

[4] C. Ariño and A. Sala, "Relaxed LMI conditions for closedloop fuzzy systems with tensor-product structure," Engineering Applications of Artificial Intelligence, vol. 20, no. 8, pp. 10361046, 2007.
[5] J. Kuti, P. Galambos, and P. Baranyi, "Polytopic TP Model based control analysis and synthesis concept," IFAC, p. accepted, 2017.

[6] G. G. Angelis, System analysis, modelling and control with polytopic linear models [Ph.D. thesis], Technische Universiteit Eindhoven, 2001, Ph.D. dissertation.

[7] J. Kuti, P. Galambos, and A. Miklós, "Output feedback control of a dual-excenter vibration actuator via GLPV model and TP model transformation," Asian Journal of Control, vol. 17, no. 2, pp. 432-442, 2015.

[8] P. Baranyi, "Output feedback control of two-dimensional aeroelastic system," Journal of Guidance, Control, and Dynamics, vol. 29, no. 3, pp. 762-767, 2006.

[9] T. T. Wang, W. F. Xie, G. D. Liu, and Y. M. Zhao, "Quasi-minmax model predictive control for image-based visual servoing with tensor product model transformation," Asian Journal of Control, vol. 17, no. 2, pp. 402-416, 2015.

[10] V. C. S. Campos, F. O. Souza, L. A. B. Torres, and R. M. Palhares, "New stability conditions based on piecewise fuzzy lyapunov functions and tensor product transformations," IEEE Transactions on Fuzzy Systems, vol. 21, no. 4, pp. 748-760, 2013.

[11] S. Chumalee and J. F. Whidborne, "Gain-scheduled $\mathrm{H}_{\infty}$ control for tensor product type polytopic plants," Asian Journal of Control, vol. 17, no. 2, pp. 417-431, 2015.

[12] P. Baranyi, L. Szeidl, and P. Várlaki, "Numerical reconstruction of the HOSVD based canonical form of polytopic dynamic models," in Proceedings of the INES 2006: 10th International Conference on Intelligent Engineering Systems 2006, pp. 196-201, gbr, June 2006.

[13] J. Kuti, P. Galambos, and P. Baranyi, "Minimal volume simplex (MVS) polytopic model generation and manipulation methodology for TP model transformation," Asian Journal of Control, vol. 19, no. 1, pp. 289-301, 2017.

[14] P. Baranyi, Y. Yam, and P. Várlaki, "Tensor Product Model Transformation in Polytopic Model-Based Control," in 1em plus 0.5em minus 0.4em Taylor, Francis Group, 4em Taylor, 2013.

[15] P. Várkonyi, D. Tikk, P. Korondi, and P. Baranyi, "A new algorithm for RNO-INO type Tensor Product model representation," in Proceedings of the INES'05: IEEE 9th International Conference on Intelligent Engineering Systems, pp. 263-266, September 2005.

[16] J. Kuti, P. Galambos, and P. Baranyi, "Non-simplex enclosing polytope generation concept for Tensor Product model transformation based controller design," in Proceedings of the 2016 IEEE International Conference on Systems, Man, and Cybernetics, SMC 2016, pp. 3368-3373, Hungary, October 2016.

[17] P. Baranyi, "Convex hull generation methods for polytopic representations of LPV models," in Proceedings of the 7th International Symposium on Applied Machine Intelligence and Informatics, SAMI 2009, pp. 69-74, Slovakia, January 2009.

[18] L. De Lathauwer, B. De Moor, and J. Vandewalle, "A multilinear singular value decomposition," SIAM Journal on Matrix Analysis and Applications, vol. 21, no. 4, pp. 1253-1278, 2000.

[19] C. Briat, Linear parameter-varying and time-delay systems, vol. 3 of Advances in Delays and Dynamics, Springer, Heidelberg, 2015.

[20] C. Eckart and G. Young, "The approximation of one matrix by another of lower rank," Psychometrika, vol. 1, no. 3, pp. 211-218, 1936.

[21] Å. Björck, "Numerics of Gram-Schmidt orthogonalization," Linear Algebra and its Applications, vol. 197, pp. 297-316, 1994.

[22] A. S. Householder, "Unitary triangularization of a nonsymmetric matrix," Journal of the ACM, vol. 5, pp. 339-342, 1958. 
[23] R. Cabrera, T. Strohecker, and H. Rabitz, "The canonical coset decomposition of unitary matrices through Householder transformations," Journal of Mathematical Physics, vol. 51, no. 8, Article ID 082101, 2010.

[24] L. N. Trefethen, "Householder triangularization of a quasimatrix," IMA Journal of Numerical Analysis (IMAJNA), vol. 30, no. 4, pp. 887-897, 2010.

[25] D. Bindel, J. Demmel, W. Kahan, and O. Marques, "On computing Givens rotations reliably and efficiently," ACM Transactions on Mathematical Software, vol. 28, no. 2, pp. 206-238, 2002.

[26] J. Pan and L. Lu, "TP model transformation via sequentially truncated higher-order singular value decomposition," Asian Journal of Control, vol. 17, no. 2, pp. 467-475, 2015.

[27] V. c. Campos, L. A. Tôrres, and R. M. Palhares, "Revisiting the TP model transformation: interpolation and rule reduction," Asian Journal of Control, vol. 17, no. 2, pp. 392-401, 2015.

[28] C. B. Barber, D. P. Dobkin, and H. Huhdanpaa, "The quickhull algorithm for convex hulls," ACM Transactions on Mathematical Software, vol. 22, no. 4, pp. 469-483, 1996.

[29] D. Avis and D. Bremner, "How good are convex hull algorithms?" in Proceedings of the the eleventh annual symposium, pp. 20-28, Vancouver, British Columbia, Canada, June 1995.

[30] J. Kuti, P. Galambos, P. Baranyi, and P. Varlaki, "A HandsOn Demonstration of Control Performance Optimization Using Tensor Product Model Transformation and Convex Hull Manipulation," in Proceedings of the IEEE International Conference on Systems, Man, and Cybernetics, SMC 2015, pp. 2609-2614, Hong Kong, October 2015.

[31] D. K. Lindner, T. P. Celano, and E. N. Ide, "Vibration Suppression Using a Proofmass Actuator Operating in Stroke/Force Saturation," Journal of Vibration and Acoustics, vol. 113, no. 4, p. $423,1991$.

[32] M. E. Polites, "New method for scanning spacecraft and balloon-borne/space-based experiments," Journal of Guidance, Control, and Dynamics, vol. 14, no. 3, pp. 548-553, 1991.

[33] R. H. Rand, R. J. Kinsey., and D. L. Mingori, "Dynamics of spinup through resonance," International Journal of Non-Linear Mechanics, vol. 27, no. 3, pp. 489-502, 1992.

[34] C.-J. Wan, D. S. Bernstein, and V. T. Coppola, "Global stabilization of the oscillating eccentric rotor," in Proceedings of the 1994 33rd IEEE Conference on Decision and Control, pp. 4024-4029, Lake Buena Vista, FL, USA.

[35] R. T. Bupp, D. S. Bernstein, and V. . Coppola, "A benchmark problem for nonlinear control design," International Journal of Robust and Nonlinear Control, vol. 8, no. 4-5, pp. 307-310, 1998. 


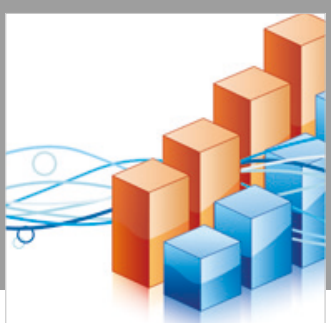

Advances in

Operations Research

\section{-n-m}
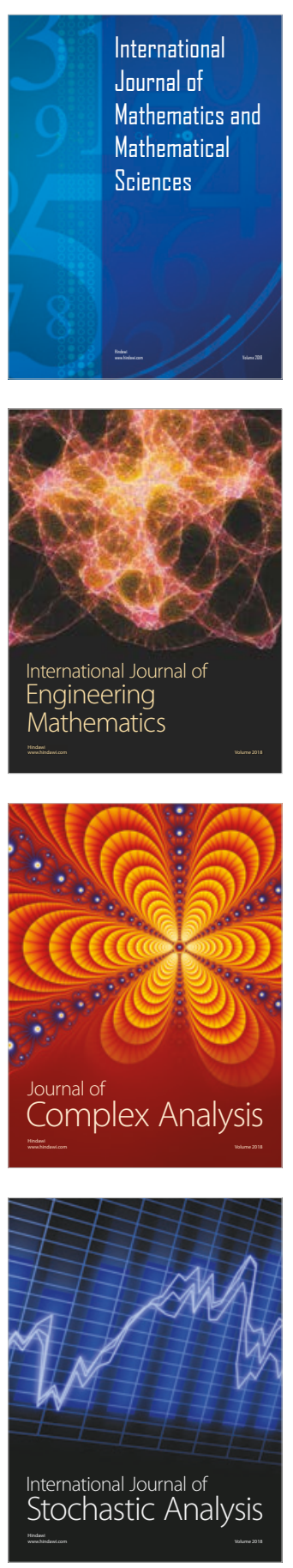
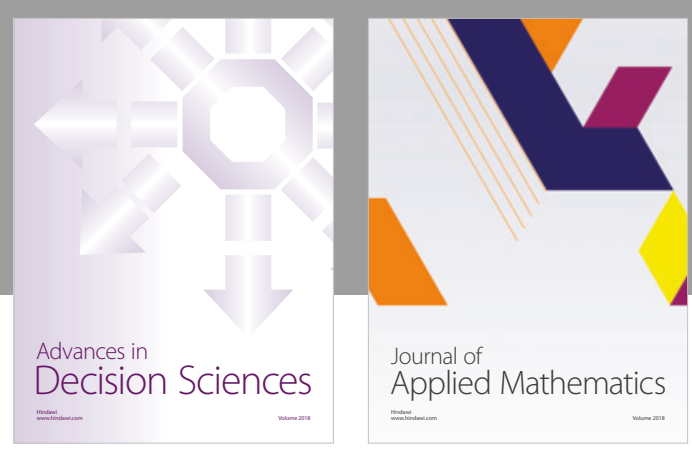

Journal of

Applied Mathematics
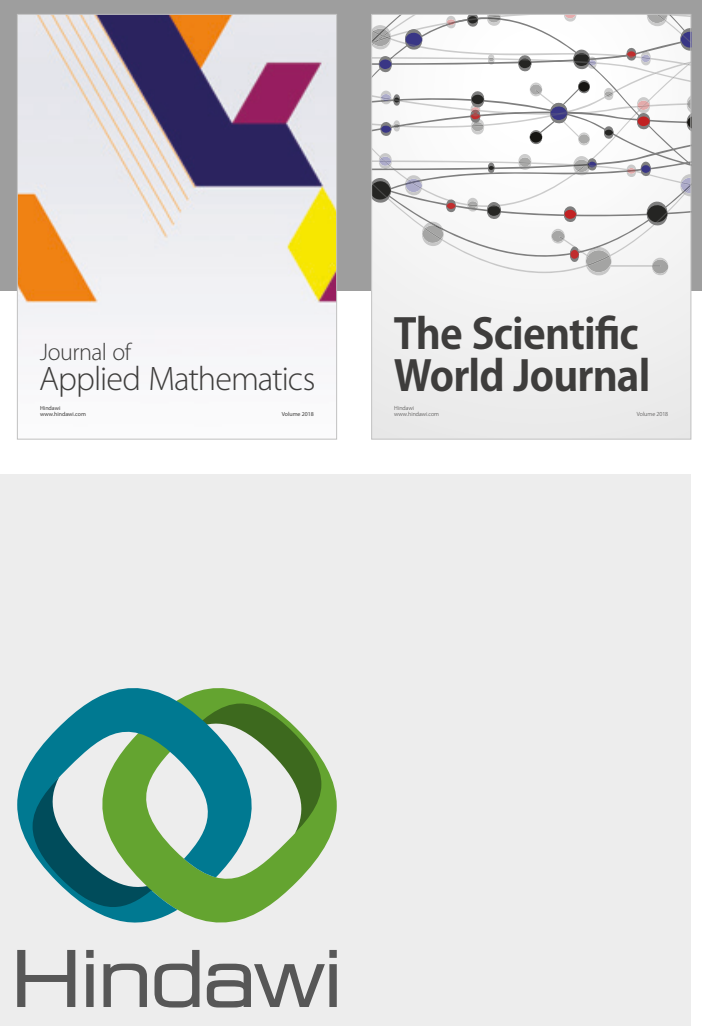

Submit your manuscripts at

www.hindawi.com

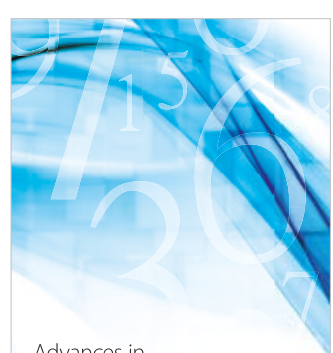

Advances in
Numerical Analysis
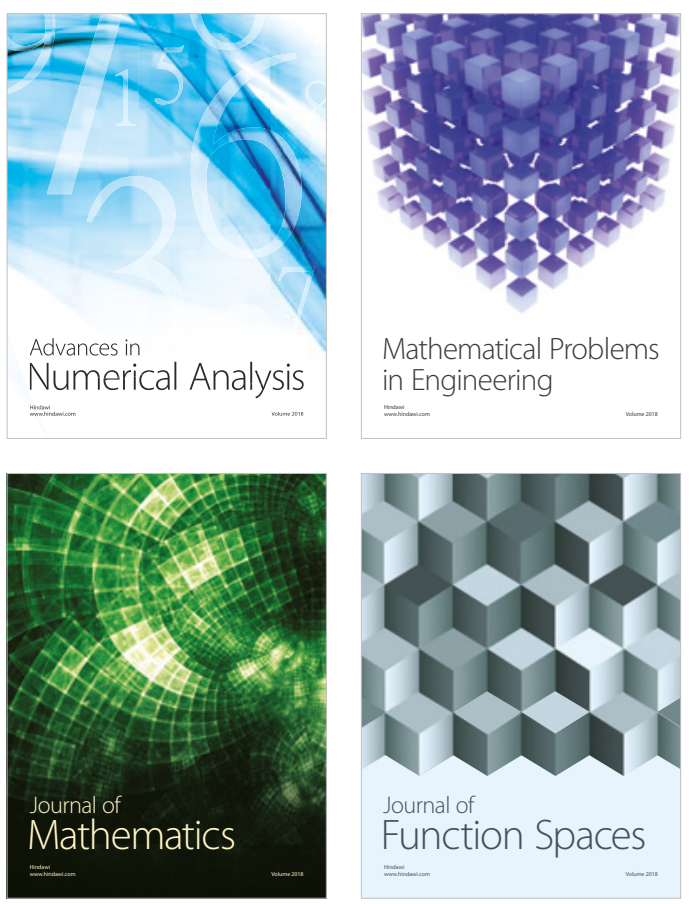

Mathematical Problems in Engineering

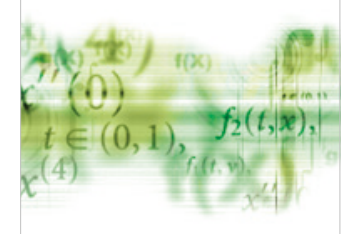

International Journal of

Differential Equations

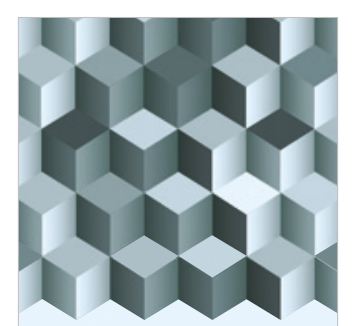

Journal of

Function Spaces
The Scientific

World Journal

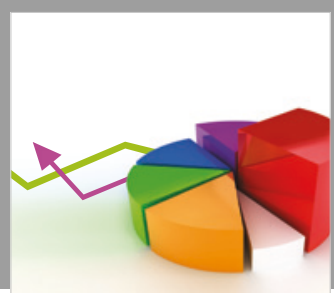

Journal of

Probability and Statistics
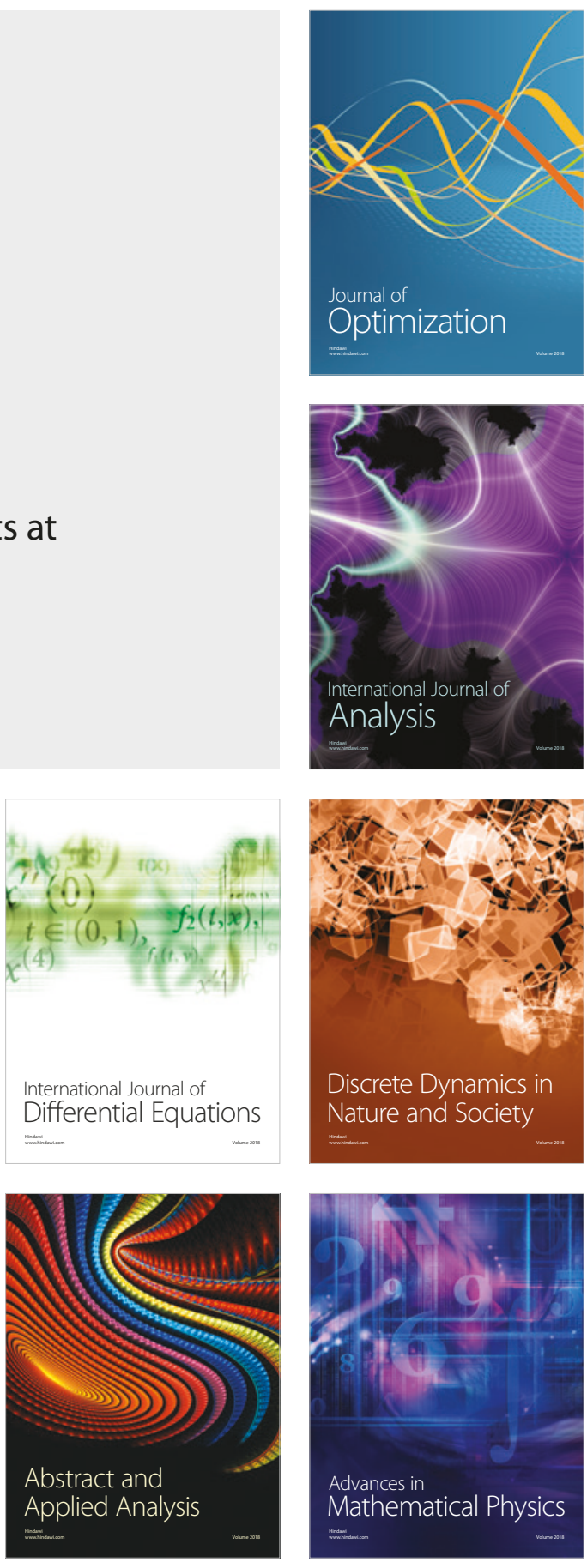